\title{
SYNAPTIC CONTROL OF EXCITABILITY IN ISOLATED DENDRITES OF HIPPOCAMPAL NEURONS ${ }^{1}$
}

\author{
LEONA M. MASUKAWA ${ }^{2}$ AND DAVID A. PRINCE ${ }^{3}$ \\ Department of Neurology, Stanford University School of Medicine, Stanford, California 94305
}

Received March 25, 1983; Revised August 4, 1983; Accepted August 16, 1983

\begin{abstract}
The apical dendrites of CA1 pyramidal cells were isolated from their cell bodies by making cuts through proximal stratum radiatum of transverse hippocampal slices from the guinea pig. This lesion separated the distal apical dendritic elements from the somata, basal dendrites, and 50 to 100 $\mu \mathrm{m}$ of the proximal apical dendritic tree. Orthodromic stimuli in stratum radiatum evoked excitatory synaptic responses in isolated dendrites, but no phasic inhibitory components could be detected. In spite of this surgically produced disinhibition, orthodromic stimuli did not elicit burst activity at the resting membrane potential. However, isolated dendrites and intact dendrites could generate multiple slow spike activity when directly stimulated with depolarizing current pulses. When isolated dendrites were depolarized by DC current, excitatory postsynaptic potentials could evoke subthreshold intrinsic slow depolarizations, or repetitive slow spikes, similar to responses elicited by depolarizing current pulses alone. After exposure to bicuculline $(5 \mu \mathrm{m})$, both intact and isolated dendrites generated bursts of activity following synaptic activation. A possible mechanism for this action of bicuculline is blockade of a residual GABA-mediated inhibition which was not expressed as a postsynaptic hyperpolarization in isolated dendrites. This bicuculline-sensitive event was capable of depressing dendritic excitability in the absence of the recurrent inhibitory synaptic input and was very effective in controlling burst activity. Our results indicate that the dendritic electrical behavior is dependent on a complex interaction between synaptic and voltage-sensitive events.
\end{abstract}

The importance of dendrites as a major site for integration of synaptic inputs in cortical neurons has been emphasized by many investigators (Andersen, 1960; see Purpura, 1967 and Llinas, 1975 for reviews). The occurrence of intrinsic (voltage-dependent) dendritic events which affect neuronal excitability has also been inferred on the basis of presumed dendritic spikes recorded with intrasomatic electrodes (Spencer and Kandel, 1961; Purpura et al., 1965) and more recently on the basis of intradendritic recordings from cerebellar Purkinje cells (Llinas et al., 1968; Llinas and Nicholson, 1971; Llinas and Hess, 1976; Llinas and Sugimori, 1980) and hippocampal pyramidal neurons (Wong et al., 1979; Wong and Prince, 1979; Schwartzkroin and Prince, 1980; Benardo et al., 1982). Information regarding the role of interac-

\footnotetext{
${ }^{1}$ This work was supported by National Institutes of Health Grant NS 12151 and the Morris Research Fund. We thank Jay Kadis for his technical assistance and Cheryl Joo for preparation of the manuscript. We are grateful to Dr. Barry Connors for his comments on the manuscript.

${ }^{2}$ Present address: Section on Neuroanatomy, Yale University School of Medicine, New Haven, CT 06510.

${ }^{3}$ To whom correspondence should be addressed.
}

tions of synaptic and intrinsic conductances of dendritic membranes in the control of neuronal excitability is incomplete. Several approaches have been used to study dendritic processing of synaptic information, as inferred from intrasomatic recordings. For example, passive cable properties of neurons have been used to establish a framework to predict events occurring at the level of the dendrite (Rall, 1967, 1977; Redman, 1973; Barrett and Crill, 1974; Traub, 1979; Traub and Llinas, 1979). Microsurgical techniques have been used in the hippocampal slice preparation to isolate local excitatory synaptic inputs to CA1 pyramidal cells in order to compare excitatory postsynaptic potentials (EPSPs) transmitted electronically to the cell soma from proximal and distal dendritic sites (Andersen et al., 1980b). Recently, by utilizing intradendritic recordings and a microsurgical technique to isolate apical dendrites (Benardo et al., 1982), we were able to study potentials generated in isolated dendrites without the interference of synaptic and voltage-dependent conductances of the cell soma. In the experiments reported here, we have utilized this preparation to examine interactions between synaptic and intrinsic events on dendritic membranes and the role of synaptic inhibition in modulating orthodromic 
excitatory responses of hippocampal pyramidal cell (HPC) dendrites.

\section{Materials and Methods}

The guinea pig hippocampal brain slice preparation was used in this study as described previously (Yamamoto, 1972; Schwartzkroin, 1975). Four hundred- to 450$\mu \mathrm{m}$ thick slices from the mid-hippocampus were superfused with an artificial cerebrospinal fluid (ACSF) containing (in mM): $\mathrm{NaCl}, 138 ; \mathrm{KCl}, 5 ; \mathrm{MgSO}_{4}, 2 ; \mathrm{CaCl}_{2}, 2$; $\mathrm{NaHCO}_{3}, 26$; and glucose, 10 . The solution was bubbled with $95 \% \mathrm{O}_{2} / 5 \% \mathrm{CO}_{2}$ to maintain a $\mathrm{pH}$ of 7.4 . In some experiments, bicuculline (Sigma) was added to the ACSF at a concentration of $5.0 \mu \mathrm{M}$. Experiments were carried out at $37^{\circ} \mathrm{C}$. Intradendritic recordings were made using $4 \mathrm{M}$ potassium acetate-filled micropipettes with resistances of 50 to 80 megohms. Penetrations of intact dendrites were made in stratum radiatum, about midway between stratum pyramidale and the hippocampal fissure. Isolated dendrites were impaled in about the same region of the middle portion of the remaining stratum radiatum. All recordings were from elements in the CA1b region (Masukawa et al., 1982).

The technique for isolation of apical dendrites of CA1 pyramidal cells from proximal apical dendrites, somata, and basilar dendrites is described in detail elsewhere (Benardo et al., 1982). In the present study, care was taken to place the razor chip used for microsurgery in stratum radiatum, about 50 to $100 \mu \mathrm{m}$ from the visible edge of stratum pyramidale. A cut was made across stratum radiatum, roughly parallel to stratum pyramidale, and this was extended at its margins to the alveolar surface, allowing physical separation of the cut piece from the remainder of the slice. This guaranteed removal of the pyramidal somata as well as the proximal apical dendritic stumps.

Recordings from isolated dendrites were made at least $1 \mathrm{hr}$ after the cut, which allowed time for presumed sealing of severed dendritic trunks. Flectrophysiological data from dendritic penetrations were accepted for analysis if the elements had resting membrane potentials $\left(V_{m}\right)$ of at least $-55 \mathrm{mV}$ and membrane input resistances $\left(R_{N}\right)$ of 30 megohms or greater. Values for $V_{m}$ and $R_{N}$, as well as the characteristics of the electrical responses of isolated dendrites and intact dendrites (generation of slow spikes, fast spikes, and inward anomalous rectification), were similar to those described previously (Wong et al., 1979; Benardo et al., 1982). Recordings were made using conventional techniques and current was passed through the microelectrode using an active bridge circuit. Bridge balance was continuously monitored and adjusted. Bipolar orthodromic stimuli were applied to stratum radiatum via a pair of sharpened tungsten wires insulated close to their tips. Stimulating electrodes were approximately $500 \mu \mathrm{m}$ from the recording site. Stimulus frequency was $0.5 \mathrm{~Hz}$ except during assessment of facilitation when the frequency was increased to $10 \mathrm{~Hz}$. The stimulus pulse duration was $0.1 \mathrm{msec}$. Data were replayed from FM tape and digitized by computer (DEC, MINC23) for analysis.

\section{Results}

Synaptic potentials of intact dendrites. Intradendritic recordings made at a level midway along the length of the apical dendrites of intact CAl pyramidal cells $(n=$ 12) often showed spontaneous EPSPs that varied in amplitude and duration (Fig. $1 A$ ). The events with the greatest rates of rise had the largest amplitudes, at times up to $2 \mathrm{mV}$; however, this relationship was not studied in detail. Although spontaneous EPSPs were recorded, spontaneous hyperpolarizing potentials were never seen.

Subthreshold postsynaptic potentials could be evoked by stimulation of stratum radiatum. The peak amplitude of the EPSP varied from 10 to $15 \mathrm{mV}$, and the inhibitory postsynaptic potential (IPSP) that followed the EPSP was smaller in amplitude, about $4 \mathrm{mV}$ (for example, see Fig. $1 B$ ). These responses were similar to those observed by Wong et al. (1979) in CA1 HPC dendrites. In contrast, EPSPs from somatic recordings of CA1 HPCs were usually less than $5 \mathrm{mV}$ in amplitude, even when relatively intense subthreshold stimuli were used and were followed by more prominent IPSPs which reached amplitudes of 5 to $10 \mathrm{mV}$ (see Masukawa and Prince, 1982). The differences in amplitude of the EPSPS and IPSPs from the intact dendrite versus the soma suggest that the EPSPs are located primarily on the dendrites and the IPSPs on the soma. Figure $1 B$ illustrates the PSP at the resting membrane potential (trace 1) and at successively hyperpolarized levels in a dendrite from an intact slice. At the resting potential the EPSP portion of the response was approximately $10 \mathrm{mV}$ in amplitude and was followed by an IPSP. As the membrane potential was hyperpolarized, the depolarizing phase of the PSP increased in amplitude (traces 2 to 5) while the IPSP was reduced (trace 2), and presumably reversed in direction (traces 3 , 4 , and 5 of Fig. $1 B$ ). The inverted IPSP summed with the shorter latency EPSP to produce a PSP with a prolonged decay lime. This effect was prominent at membrane potentials hyperpolarized by $40 \mathrm{mV}$ from rest $(\sim-100 \mathrm{mV})$, i.e., negative to the reversal potential of the IPSP (Andersen et al., 1980b; Dingledine and Gjerstadt, 1980), as is shown in trace 5 of Figure $1 B$.

Increasing the orthodromic stimulus strength increased the EPSP amplitudes until threshold was reached and a spike was generated (cf. Fig. $1 C$, traces 1 , 2 and 3,4). Partial spike responses were also occasionally evoked (Wong et al., 1979). Increasing the stimulus strength also increased the rate of rise of the EPSP and increased the amplitude of the following hyperpolarization to a maximum of approximately $10 \mathrm{mV}$ beyond the resting membrane potential. For example, the slopes of the rising phases of the subthreshold EPSPs in Figure $1 C$, traces 1 and 2 are $3.7 \mathrm{~V} / \mathrm{sec}$ and $9.8 \mathrm{~V} / \mathrm{sec}$, respectively. The IPSPs following responses of trace 1 and trace 2 reach peak amplitudes of $4.4 \mathrm{mV}$ and $10 \mathrm{mV}$, respectively. Under normal conditions, bursting was never evoked in recordings from intact dendrites during orthodromic stimulation.

Synaptic potentials in isolated dendrites. After the pyramidal cell somata, basilar dendrites, and proximal apical dendrites were removed, microelectrode penetrations 
A
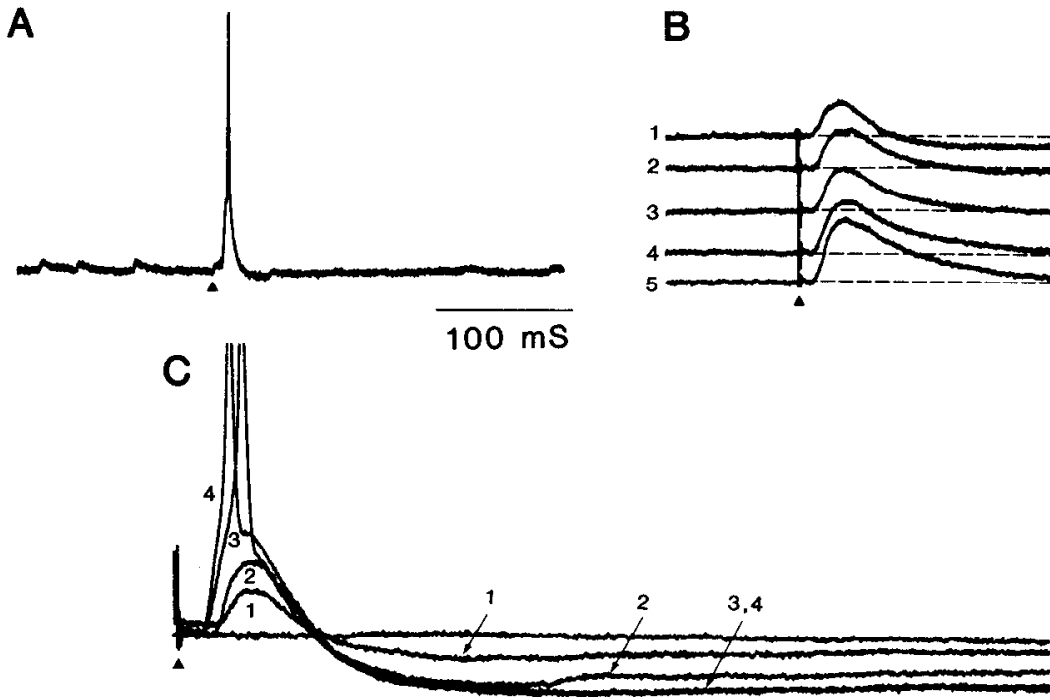

B

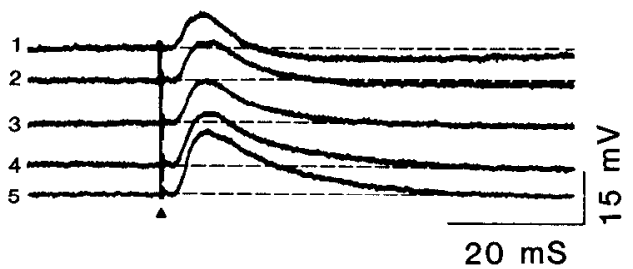

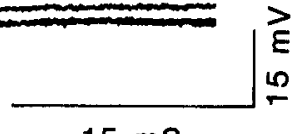

$15 \mathrm{mS}$

Figure 1. Intradendritic synaptic responses from intact CA1 hippocampal pyramidal neurons. $A$, Spontaneous EPSPs (first three events of trace) had rapid rising phases and decayed more slowly. Orthodromic stimulus (arrowhead in this and subsequent figures) evoked an EPSP and spike, followed by a small hyperpolarization and decrease in spontaneous EPSP amplitude. Resting $V_{m}=-68 \mathrm{mV}$. $B$, Postsynaptic potentials in dendrite of $A$ evoked by a constant orthodromic stimulus at varying membrane potentials. Trace 1, EPSP-IPSP sequence generated at resting $V_{m}$. Dashed line, base line potential for each trace. In traces 2 to 5 , responses were evoked as the $V_{m}$ was held at increasingly more negative levels with DC current. Position of traces reflects imposed change in $V_{m}$. $C$, Orthodromic stimulus strength was increased in steps, with $V_{m}$ at resting level. The first stimulus (trace 1) generated an EPSP-IPSP sequence. As the stimulus was increased in strength (trace 2), both the EPSP and IPSP components of the synaptic response increased in amplitude. More intense stimuli (trace 3) evoked EPSPs suprathreshold for generation of fast spikes, but only a single spike could be evoked by even the most intense stimulus (trace 4). Large hyperpolarizations followed more intense sub-and suprathreshold stimuli. Voltage calibration in $B$ for traces $A$ and $B$.

were made within the remaining segments of the apical dendrites $(n=16)$ using techniques identical to those employed for penetration of intact dendrites. Spontaneous EPSPs were present, but their frequency was not decreased after an orthodromically evoked EPSP (cf. Figs. $1 A$ and $2 A$ ). In isolated, as in intact, dendrites, spontaneous IPSPs were never observed. The PSPs which could be evoked orthodromically were different in nature from those of intact dendrites. At resting membrane potential, stimulus intensities which were subthreshold for spike generation evoked a pure depolarizing wave without a sign of a longer latency hyperpolarizing PSP (Fig. 2B, trace 1). As the membrane potential was hyperpolarized, the PSP increased in amplitude and rate of rise; however, the PSP duration was not increased (cf. Figs. $1 B$ and $2 B$ ), suggesting that an inverted IPSP was not present. In contrast to the findings in intact dendrites (Fig. 1C), increasing the orthodromic stimulus intensity did not evoke large hyperpolarizing PSP components in isolated elements (Fig. $2 C$ ). As the stimulus intensity was increased, the EPSP increased in amplitude and sometimes in duration. The four superimposed sweeps of Figure $2 C$ are dendritic responses to increas- ingly intense orthodromic stimuli. The weakest stimulus evoked an EPSP of about $8 \mathrm{mV}$, with a slow falling phase (Fig. 2C, trace 1). A more intense, but subthreshold, stimulus evoked a large amplitude, faster rising EPSP which was prolonged by a slow depolarizing potential (Fig. 2C, trace 2, arrow). Further increases in stimulus intensity evoked faster rising EPSPs which triggered fast spikes (Fig. 2C, traces 3 and 4 ). Increases in the stimulus intensity to still higher levels, supramaximal for spike generation, failed to evoke a hyperpolarizing component of the response (cf. Figs. $1 C$ and $2, C$ and $D$ ). Such intense orthodromic stimuli did, however, elicit additional depolarizing responses. In Figure $2 D$ the least intense stimulus evoked an EPSP which triggered a fast spike (trace 1). Progressive increases in stimulus intensity evoked a slow depolarizing response which followed the spike (Fig. $2 D$, traces 2 and 3 ). This depolarization could continue to increase in amplitude over many milliseconds and lead to the generation of a high threshold slow spike (Fig. $2 D$, trace 4 ) which was similar in appearance to the $\mathrm{Ca}^{++}$-mediated spikes previously described in CA1 and CA3 HPC dendrites (Wong et al., 1979; Benardo et al., 1982). High threshold slow spikes 
A
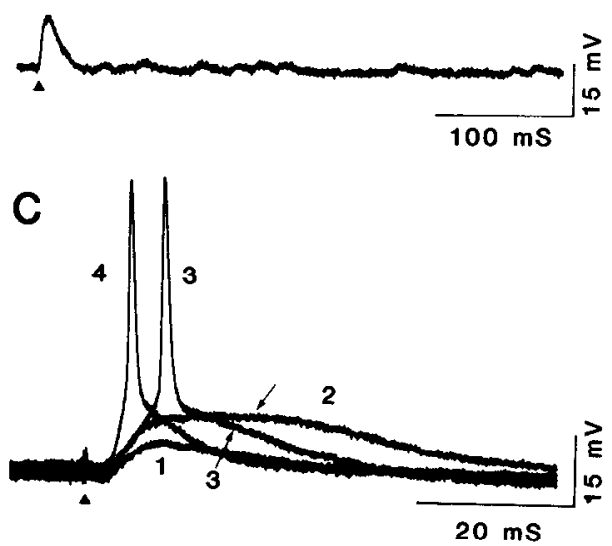

B

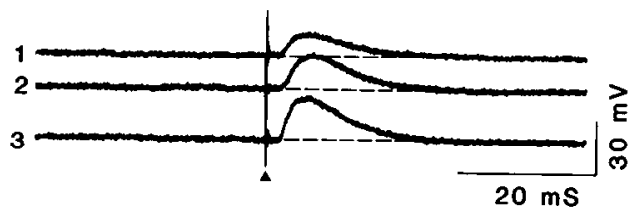

D

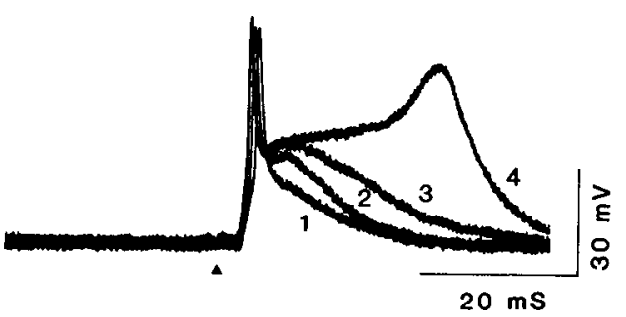

Figure 2. Intradendritic synaptic responses from isolated dendrites. A, Orthodromically evoked EPSP is followed by spontaneous EPSPs. $B$, PSP from an isolated dendrite evoked at resting $V_{m}$ (trace 1 ) and at hyperpolarized $V_{m}$ (traces 2 and 3) consists only of a depolarizing response whose duration is unaffected by $V_{m}$. The dashed lines are extensions of the base lines. Resting $V_{m}=-65 \mathrm{mV}$. Position of traces 2 and 3 reflects actual changes in $V_{m}$. $C$, Responses of the same dendrite to orthodromic stimuli of increasing intensity. Low-intensity stimuli evoked simple EPSPs (trace 1). Responses to stimuli of increasing intensity are shown in traces 2 to 4 . Response of trace 2 was prolonged by a large superimposed depolarizing hump (arrow). EPSP rise time decreases as stimulus intensity increases. $D$, Isolated dendrite generates a slow depolarizing potential following each orthodromically evoked spike (traces 1,2, and 3). The most intense stimulus (trace 4) elicited a depolarizing potential which increased slowly in amplitude to evoke a slow spike.

were seen only infrequently in orthodromically stimulated isolated dendrites.

Membrane time constant and the decay time constant of the EPSP. That IPSPs were not evident in isolated dendrites was further supported by comparing the time course of the falling phase of the EPSP evoked orthodromically in intact and isolated dendrites with that of the membrane electrotonic response to a small hyperpolarizing current pulse. As shown in Figure $3 A$ in intact dendrites, the time constant of the membrane, obtained by plotting the log of the membrane potential change to current versus time, was slower than that of the decay phase of the EPSP. This was presumably due to the contribution of the IPSP-associated hyperpolarization to the falling phase of the EPSP. In isolated dendrites (Fig. $3 B$ ) the time constant of the falling phase of the EPSP was greater than the membrane time constant. 4 These findings, together with those of Figure 2, suggest that at least the major component of the IPSP, which is usually a part of the orthodromic response in intact dendrites, is eliminated by removing the soma and proximal 50 to 100 $\mu \mathrm{m}$ of the apical dendrite. An increase in the time constant of the decay phase of the EPSP has also been reported in recordings from intact CA1 HPC dendrites (Wong and Prince, 1979) and somata (Dingledine and

\footnotetext{
${ }^{4}$ Possible explanations for an EPSP decay time constant being slower than the membrane time constant include the occurrence of a slow intrinsic membrane response during the repolarizing phase of the EPSP (e.g., Fig. 2C, arrow) even with "subthreshold" stimuli; an electrotonic reflection of electrically remote EPSPs; a prolonged and slowly resolving EPSP conductance change; or an additional depolarizing excitatory or inhibitory synaptic potential.
}

Gjerstad, 1979, 1980; Schwartzkroin and Prince, 1980) exposed to penicillin, as a consequence of blockade of postsynaptic inhibition by this agent. Under conditions of pharmacological disinhibition, orthodromic stimuli become effective in evoking synchronous epileptiform depolarization shifts and spike bursts in intact HPCs (Wong and Prince, 1979; Schwartzkroin and Prince, 1978, 1980; Dingledine and Gjerstad, 1980). Although inhibition in the present experiments had been either greatly reduced or removed entirely, events of this sort were not readily evoked orthodromically in isolated dendrites.

Effects of facilitation on PSPs. A further reflection of the loss of the IPSP in isolated dendrites was noted during the first $10 \mathrm{sec}$ of a high-frequency (e.g., $10 \mathrm{~Hz}$ ) orthodromic stimulus. In intact dendrites the depolarizing phase of the PSP increased in amplitude during such stimulation concomitant with an increase in resting membrane potential and elimination of the hyperpolarizing component of the PSP (cf. traces 1 and 2 of Fig. $4 A$ ). The membrane potential hyperpolarized steadily in response to repetitive stimuli, to a plateau level close to the IPSP reversal potential, which in these neurons is 5 to $10 \mathrm{mV}$ below resting potential, or about -65 to -75 $\mathrm{mV}$ (Andersen et al., 1980b; Dingledine and Gjerstadt, 1980 ), and returned to the prestimulus level after stimulation was stopped. A similar change in membrane potential during the initial period of a tetanic stimulation also occurs in motoneurons (Llinas et al., 1974). The apparent increase in the amplitude of the depolarizing phase of the PSP after 20 stimuli shown in Figure $4 A$ could then be due in part to the shift in the resting 
A

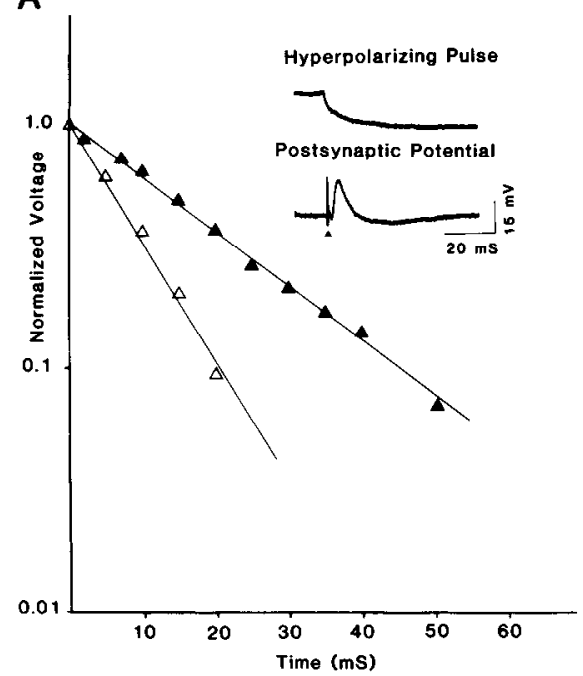

B

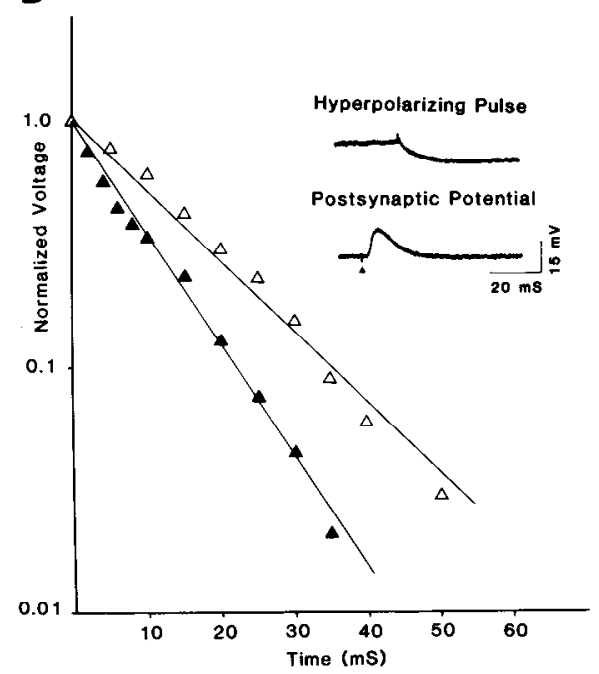

Figure 3. Comparison of membrane charging curves and falling phases of PSPs in intact and isolated dendrite. $A$, Voltage response of an intact dendrite to hyperpolarizing current step (inset) was plotted semilogarithmically $(\boldsymbol{A})$. The voltage decay from the peak of the depolarizing phase of the PSP from the same cell (inset) was plotted in the same way $(\triangle)$. The time constant of the electrotonic response was slower than that of the decay phase of the PSP, presumably due to onset of the IPSP on falling phase of the EPSP. $B$, Both the decay phase of the PSP $(\triangle)$ and the charging response to a hyperpolarizing current step (A) from an isolated dendrite were plotted as in $A$. The voltage responses used for these plots are shown in the inset. Note that the decay of the PSP has a time constant that is slower than the membrane time constant. Lines in plots of $A$ and $B$ were drawn by eye from normalized voltage values. The early fast decay component of the electrotonic membrane response was eliminated in these plots.
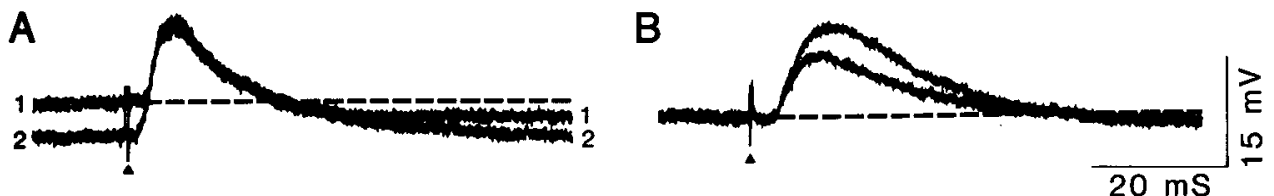

Figure 4. Responses of intact $(A)$ and isolated $(B)$ dendrites during $10-\mathrm{Hz}$ orthodromic stimulation. A, trace 1, Initial PSP of the train. Trace 2, Response to twentieth stimulus. The base line hyperpolarized, and the EPSP became larger in amplitude. The base line recovered to prestimulus levels following cessation of stimulation (not shown). $B$, In an isolated dendrite, responses obtained under the same conditions show that the EPSP increases in amplitude during the $10-\mathrm{Hz}$ stimulus train, without a hyperpolarizing shift in the membrane potential. The time to peak amplitude also increased after the short $10-\mathrm{Hz}$ stimulus. Dashed lines, base line $V_{m}$ for first response of train in $A$ and $B$.

potential away from the EPSP equilibrium potential (since absolute level of the EPSP peak amplitudes did not change) and to presynaptic facilitation. The shift in membrane potential in intact dendrites appeared to be due to the summation of IPSP voltage changes since it was blocked in the presence of bicuculline (not shown).

A slightly different effect of repetitive stimulation on the PSP amplitude was seen in isolated dendrites. The membrane potential did not shift during repetitive stimulation, although the PSP amplitude was increased. This response was a clcar cxample of synaptic facilitation (Fig. $4 B$ ). Neither before nor during repetitive stimulation did the membrane potential hyperpolarize following the EPSP (cf. Fig. 4, $A$, trace 1, and $B$ ). As judged by the lack of shift in the $V_{m}$ during repetitive stimulation, there appeared to be no definite signs of summated hyperpolarizing IPSPs in isolated dendrites.
Orthodromically evoked, potential dependent dendritic responses. Although an IPSP was not evident as a hyperpolarization following an EPSP in isolated dendrites (Fig. 2, $B, C$, and $D$ ), it was still possible that these elements might possess an inhibitory potential whose reversal level was at or positive to the resting potential, either due to a shift in the chloride equilibrium potential or the presence of a depolarizing EPSP. In order to investigate this point and to study the potential dependent conductances triggered by PSPs, EPSPs which were subthreshold at the resting potential were evoked in isolated dendrites while the membrane was depolarized to various levels. Typical responses are shown in Figure 5. Small depolarization (approximately $5 \mathrm{mV}$ positive to resting membrane potential) did not uncover a hyperpolarizing component following the EPSP (Fig. 5A, traces 2 and 3 ). As the membrane was further depolar- 


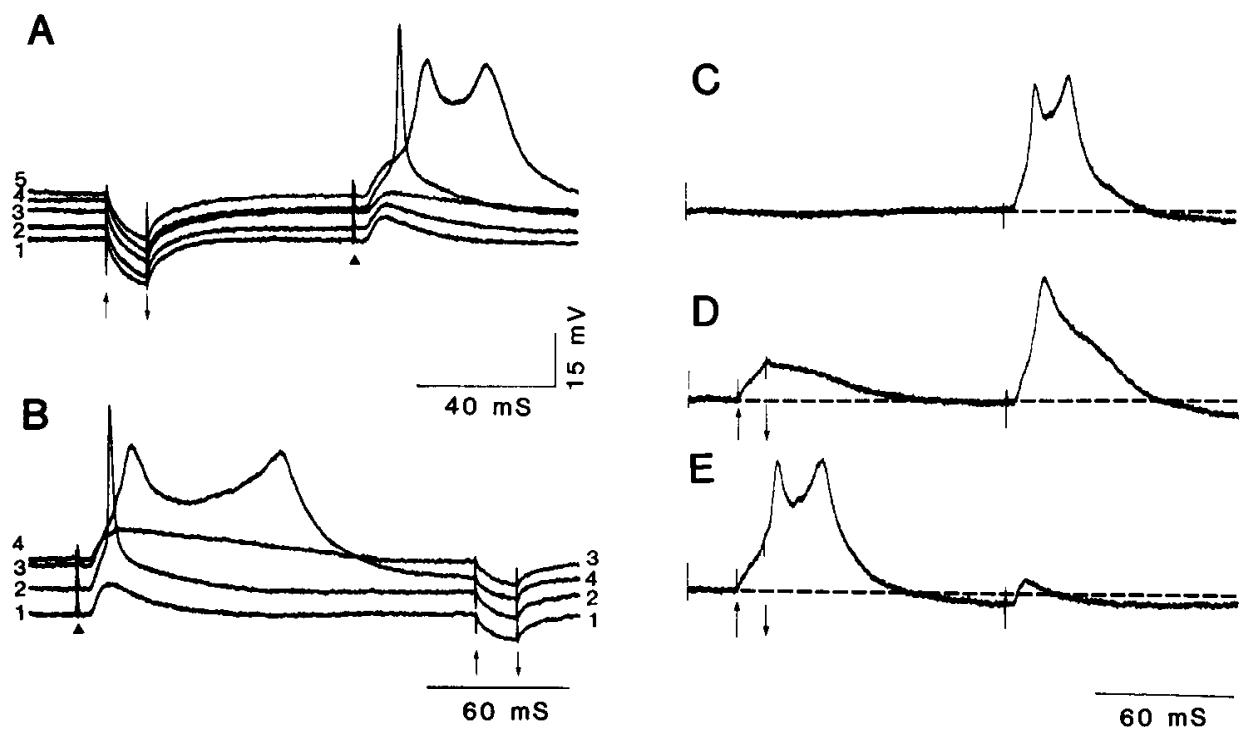

Figure 5. $A$ and $B$, The interaction of EPSPs and membrane depolarization in isolated dendrite. $A$, Responses to orthodromic stimuli of constant amplitude at resting $V_{m}$ (trace 1 ) and progressively depolarized $V_{m}$ s (traces 2 to 5 ). In each sweep a response to a hyperpolarizing constant current pulse (arrows) precedes the orthodromic stimulus (arrowhead). Traces 1 to 3, Hyperpolarizing synaptic component does not develop during small membrane depolarizations. Trace 3, Membrane depolarization results in an apparent increase in the duration of the EPSP. Trace 4, EPSP evokes a single fast spike. Trace 5, EPSP evokes two slow spikes. $B$, Orthodromic responses to constant stimuli in same isolated dendrite at different membrane potentials. Trace 1, At resting $V_{m}$, EPSP alone is evoked. Trace 2, With small depolarization, EPSP evokes fast spike. Trace 3, With further depolarization, EPSP evokes slow depolarizing event. Trace 4, With slight additional depolarization, EPSP evokes prolonged depolarization consisting of two slow spike potentials. Responses to brief hyperpolarizing current pulses (arrows) which follow orthodromic responses show increased conductance during the afterhyperpolarization following response of trace 4. $C$ to $E$, Responses of the same dendrite to depolarizing current pulses (arrows) and orthodromic stimuli. Dendrite was depolarized with DC current to $V_{m}$ level of $A$, trace 5. $C$, No current step applied; orthodromic stimulus elicits EPSP which evokes slow depolarization and two slow spikes. $D$, Current pulse evokes slow depolarization, and succeeding orthodromic stimulus evokes a single slow spike with prolonged depolarizing afterpotential. $E$, More intense depolarizing current pulse evokes slow depolarization containing two slow spikes and several inflections followed by afterhyperpolarization. Orthodromic stimulus falling during afterhyperpolarization evokes EPSP. Time calibration for traces of $C$ to $E$ is shown under $E$; voltage calibration for all traces is shown under A.

ized, a slow, large-amplitude depolarizing wave and fast spike were evoked by the orthodromic stimulus (Fig. $5 \mathrm{~A}$, trace 4). These events might have obscured a hyperpolarizing component of the PSP had it been present. At a still more depolarized membrane potential, orthodromic stimuli evoked a larger amplitude depolarizing event which reached threshold for eliciting two slow spikes (Fig. $5 A$, trace 5). If orthodromic stimuli did evoke an IPSP in this dendrite, the IPSP was not expressed as a hyperpolarization during small membrane depolarizations, nor was it powerful enough to block slow spike activity and prolonged slow potentials once the EPSP was raised above threshold by DC membrane depolarization.

The dendritic responses of Figure $5 B$ illustrate another important feature of synaptic activation not previously appreciated. In the absence of feedback inhibition, orthodromic excitation by a constant intensity stimulus can have several consequences, depending upon $V_{m}$. At the resting membrane potential a subthreshold EPSP is evoked (Fig. $5 B$, trace 1). When the membrane is depolarized from rest, the EPSP may trigger a fast spike (Fig. $5 B$, trace 2). EPSPs evoked at depolarized membrane potentials also may elicit an additional intrinsic slow depolarizing event which sums with the EPSP, as is clearly seen in Figure $5 B$, trace 3. If the orthodromic stimulation falls at still more depolarized levels, the threshold for slow, presumed $\mathrm{Ca}^{++}$-mediated spikes may be reached (Fig. $5 A$, trace 5 , and $B$, trace 4 ).

The intrinsic slow depolarizations evoked by the EPSP in the dendrite of Figure $5 B$, trace 3 are illustrated more clearly in Figure 6. Traces $A$ and $B$ show orthodromic EPSPs evoked by the same intensity stimulus at the resting membrane potential level $(B)$ and at a membrane potential about $5.5 \mathrm{mV}$ depolarized from the resting potential $(A)$. The EPSP at the more positive membrane potential has a slower falling phase; the difference between these two responses is seen in the subtracted trace of Figure $6 A-B$. When the orthodromic stimulus intensity is increased slightly, a larger amplitude, faster rising 

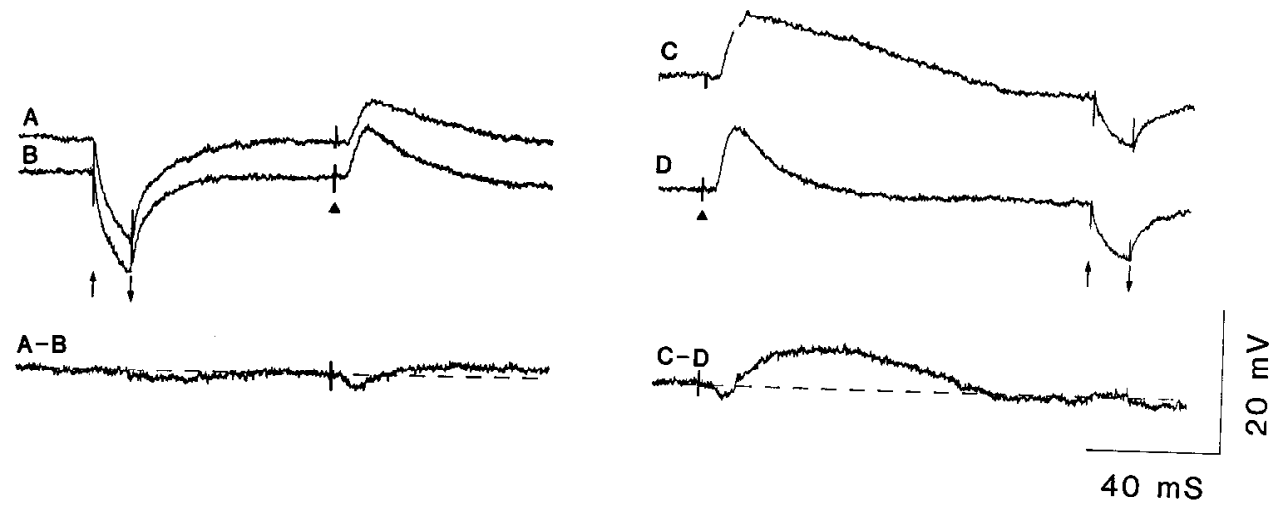

Figure 6. Orthodromic responses at resting $V_{m}(B$ and $D)$ and at $V_{m} \mathrm{~s}$ about $5.5 \mathrm{mV}(A)$ and $15 \mathrm{mV}(C)$ depolarized from rest in dendrite of Figure 5. Subtracted traces $A-B$ and $C$ $D$ show that the EPSP evokes a slow subthreshold depolarization at the depolarized $V_{m}$. Initial hyperpolarization (seen in $A-B$ and $C-D$ ) is discussed in the text. Orthodromic stimulus intensity is the same in $A$ and $B$. Stimulus intensity is the same in trace $C$ as $D$, but it is increased over that in $A$ and $B$. Hyperpolarizing responses produced by current pulses (arrows) show no significant changes in $R_{N}$ during depolarization from $A$ and $B$.

EPSP can be evoked at the resting potential $(D)$. If the stimulus of $D$ is delivered while the membrane potential is depolarized with DC current to a level about $15 \mathrm{mV}$ positive to rest (trace $C$ ), a prolonged depolarizing event is evoked by the EPSP. The subtracted response of Figure $6 C-D$ shows that the EPSP elicits a slow depolarization with a peak amplitude of about $5 \mathrm{mV}$ which lasts about 80 msec. It appears from the data of Figure 6 that the EPSP-evoked slow depolarization may be graded in amplitude (cf. Fig. 6, $A-B$ and $C-D$ ) and dependent on the membrane potential level and the amplitude of the superimposed EPSP. The slow synaptically evoked depolarization of Figure $6 C$ fails to evoke a fast spike, even though its peak is well above threshold (cf. traces 2 and 3 of Fig. $5 B$ ), perhaps because of inactivation of the spike conductance at this level of depolarization. A slight hyperpolarization preceding the slow depolarization is present in the subtracted responses of Figure $6, A-B$ and $C$ $D$. The hyperpolarization appears to be a consequence of the slightly slower rate of rise of the EPSP at depolarized potentials, which affects the subtracted responses. These differences in EPSPs may be due to an underlying IPSP that is made obvious by the increase in driving force during the membrane depolarization or to the shift of EPSPs toward their equilibrium potential which occurs when the membrane potential is depolarized. We are not able to distinguish between these possibilities. The changes in EPSP voltage and time course during depolarization are not due to apparent changes in $R_{N}$ since significant alterations in $R_{N}$ were not seen over the same range of membrane potentials (Figs. $5 A$ and $6, A, B$, and $A-B)$. These intrinsic slow depolarizations are similar to those noted during intense orthodromic stimulation at resting potential in some dendrites (Fig. $2 C$, arrow). In control recordings from intact dendrites, we noted no EPSP-evoked slow responses, presumably because of the action of the orthodromically evoked IPSPs which block both intrinsic slow depolarizations and slow spike and burst responses (see also Wong and Prince, 1979).

Similar intrinsic responses could be directly elicited by applying depolarizing current pulses. In the examples of Figure 5, $D$ and $E$ from a dendrite which was depolarized to facilitate intrinsic responses, the first response was evoked by a short depolarizing current pulse (arrows) which elicited either a slow subthreshold depolarizing afterpotential $(D)$ or, at higher intensity, a larger depolarization containing two slow spikes $(E)$. Figure $5, D$ and $E$ also shows that the response to subsequent orthodromic stimuli were modified by the alterations in membrane potential and/or presumed conductance increases which followed the preceding directly evoked events. Figure $5 C$ illustrates that the control orthodromic response consisted of a slow depolarization and two slow spikes. When the conditioning current pulse elicited a slow depolarizing wave, the succeeding orthodromic stimulus produced a single slow spike and depolarizing afterpotential (Fig. 5D). A more intense conditioning current pulse (Fig. $5 E$ ), which evoked a burst of two slow spikes, was followed by an afterhyperpolarization (AHP). The orthodromic response which fell during the AIIP consisted of a discrete EPSP of moderate amplitude (second response of Fig. $5 E$ ). Orthodromically evoked responses containing slow spikes were also regularly followed by AHPs and conductance increases (see Fig. $5 B$, trace 4). By contrast, simple EPSPs (Fig. $5 B$, trace 1) and EPSPs which evoked a single fast spike (Fig. $5 B$, trace 2) were not followed by significant AHPs or conductance increases.

Effects of bicuculline on dendritic responses. From the data presented above, it appears that inhibitory components of the orthodromic response were largely removed by the cutting procedure, and yet at normal resting membrane potentials isolated dendrites did not generate bursts of spikes following intense suprathreshold orthodromic stimuli. This finding appeared to contradict previous results which suggested that disinhibition allows orthodromic EPSPs to trigger intrinsic burst discharges (Wong and Prince, 1979). However, these previous experiments employed a general pharmacological blockade of inhibitory synaptic input. It seemed possible that functional inhibition might still be present in the isolated dendritic preparation due to a small but well localized 
synaptic input. In order to test this possibility, we bathed intact slices and those with isolated dendrites in ACSF containing $5 \mu \mathrm{M}$ bicuculline. Orthodromically evoked responses following such treatment are shown in Figure 7. A weak stimulus could evoke a small amplitude EPSP which had a long duration (trace 1 of Fig. 7, $A$ and $B$ ). Small increments in stimulus intensity evoked responses composed of multiple spikes. These responses were generated in both intact and isolated dendrites and were made up of short latency fast spikes and subsequent slow spikes (Fig. 7, $A$ and $B$, traces 2 and 3 ). The spike events in intact dendrites were overlapping and included many partial spikes with multiple inflections, perhaps reflecting the electrotonic invasion of spikes from the intact cell body and axon hillock or other dendritic sites (Fig. $7 A$, traces 2 and 3 ). These small overlapping spikes were not present in the isolated dendrites. The responses to orthodromic stimulation in intact dendrites exposed to bicuculline were similar to those seen by Wong and Prince (1979). It was not possible to generate an EPSP of more than 5 to $10 \mathrm{mV}$ without also generating a spike and slow depolarizing events. The responses shown in Figure 7 were obtained at resting potentials comparable to those of elements in normal ACSF; orthodromic bicuculline burst responses did not require DC depolarization of the membrane. Others have shown that bicuculline can directly affect membrane properties of cultured mouse spinal cord neurons at threshold concentrations much higher than used here (Heyer et al., 1982). We noted no large differences in resting membrane potential or $R_{N}$, measured from responses to hyperpolarizing current pulses, between bicuculline and control groups. However, we were unable to study single dendrites before and after exposure to the drug. Data avail- able for penicillin, another GABA blocker, also suggest that significant changes in $R_{N}$ are not produced when low concentrations, sufficient to initiate synchronous burst activity, are applied to hippocampal slices (Schwartzkroin and Prince, 1978; Dingledine and Gjerstad, 1980; Hotson and Prince, 1981). Therefore, it seems probable that the effect of bicuculline in promoting burst activity was through block of GABA receptors rather than through an effect on the nonsynaptic dendritic properties.

\section{Discussion}

The microdissection technique used in these experiments physically eliminated a major portion of the inhibitory input to the CA1 pyramidal cells through removal of the soma, proximal one-fourth of the apical dendrites, and the entire basilar dendritic region. From morphological studies, $95 \%$ of the symmetric synapses that contain flattened vesicles and are presumed to be inhibitory are located on the pyramidal cell soma and on the dendritic trunks approximately $80 \mu \mathrm{m}$ to either side of stratum pyramidale (Blackstad and Flood, 1963). The source for the feedback inhibitory current as determined from field potential recordings is also located at the layer of the pyramidal soma (Andersen et al., 1966). Data from localized application of GABA show that the hyperpolarizing membrane response is mainly associated with the soma and that dendritic applications produce depolarizations (Alger and Nicoll, 1979; 1982b; Andersen et al., 1980a; but see Wong and Watkins, 1982, and below). These depolarizing responses are believed to be extrasynaptic because they are not produced by the specific GABA analogue, 4,5,6,7-tetrahydroisoxazolo-[5,4-C]pyridine-3-ol (THIP), and are seen under specific phar-

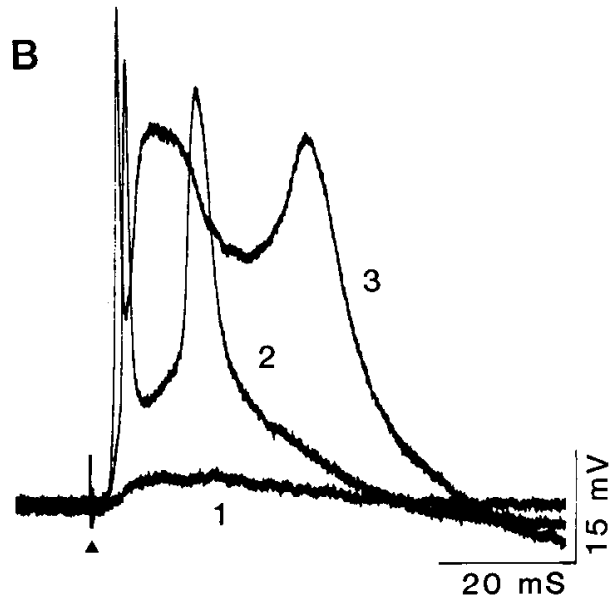

Figure 7. Responses of intact $(A)$ and isolated $(B)$ dendrites during orthodromic stimulation after exposure to bicuculline $(5 \mu \mathrm{M})$. $A$, An intact dendrite generated a subthreshold EPSP with slow falling phase at a low stimulus intensity (trace 1). A slight increase in stimulus intensity evoked a faster rising, slightly larger EPSP which triggered spikes with several inflections and a slow depolarization (trace 2). Further increase in stimulus intensity evoked a more prolonged burst with multicomponent faster and slower spikes (trace 3). B, An isolated dendrite recorded at resting membrane potential generated a small EPSP when subthreshold orthodromic stimuli were applied (trace 1). Increases in stimulus intensity could evoke slow depolarizations with fast and slow spike components which became longer in duration as intensity increased (lraces 2 and 3 ). All responses are shown at the resting membrane potential. Spikes are amputated in sweeps of $A$. 
macological conditions (Alger and Nicoll, 1982b). Therefore, there is strong circumstantial evidence that in CA1 pyramidal cells thr majority of the inhibitory synaptic input is localized on the soma and proximal apical dendrites, which we have removed by the microsurgery, and that inhibitory synaptic input to the apical dendrite, if any, normally would be hyperpolarizing.

It has been previously proposed that the feedback or recurrent inhibitory postsynaptic input described above is ir.portant in preventing the pyramidal cell from firing $\mathrm{r}$. Jre than one spike following intense orthodromic stimali (Andersen et al., 1963, 1964). On this basis, we would have expected that removal of the recurrent inhibition by a microsurgical procedure would give rise to a population of elements which would generate burst discharges following orthodromic excitation. However, our data indicate that, even though there were no obvious signs of phasic hyperpolarizing IPSPs, isolated dendrites were still incapable of generating orthodromically evoked slow depolarizations and repetitive spikes except on rare occasions when high-intensity stimuli were applied (e.g., Fig. 2D). After exposure to bicuculline, however, the isolated dendrites generated burst discharges following low-intensity orthodromic stimuli-a situation similar to that seen in intact neurons under the same conditions. The most likely explanation for this finding is the persistence of a residual inhibitory synaptic influence on the isolated dendritic elements. This presumably amounts to only about $5 \%$ of the total inhibitory input to a pyramidal cell (Gottlieb and Cowan, 1972) but, nonetheless, is functionally very effective in suppressing synaptically triggered intrinsic slow depolarizations at the level of the dendrites. Interneurons are present throughout stratum radiatum (Lorente de No, 1934), and glutamic acid decarboxylase (GAD) (Storm-Mathisen and Fonnum, 1971) and somata containing GAD (Ribak et al., 1978) are present in the apical dendritic region. The observations that dendritic applications of small amounts of GABA (Wong and Watkins, 1982), or the specific analogue THIP (Alger and Nicoll, 1982b), produce hyperpolarization indicate that appropriate inhibitory receptors are present there and are highly localized. Significant inhibitory influences have been reported on dendrites of CA1 hippocampal pyramidal cells of anesthetized rabbit (Fujita, 1979) and rat (Leung, 1978), as well as on dendrites of cerebellar Purkinje cells (Llinas et al., 1968; Llinas and Nicholson, 1971). These data taken together would lead to the expectation that direct inhibitory actions at the dendritic level should be present and hyperpolarizing in polarity. Alger and Nicoll (1982a), on the basis of somatic recordings, have also suggested a feedforward inhibition at the level of CA1 pyramidal cell dendrites.

'The explanation for prominent bicuculline effects in the absence of an obvious hyperpolarizing IPSP or conductance change on isolated dendrites is not obvious from our experiments. We have considered the possibility that bicuculline might produce a direct effect on the excitability of dendritic membrane, since the drug is known to produce decreases in $\mathrm{K}^{+}$conductance (Heyer et al., 1982). However, these effects occur at much higher concentrations than were used in our experiments. An- other possible mechanism for the bicuculline effects might be blockade of a GABA-mediated presynaptic inhibition which normally maintains EPSP amplitude below that necessary for activation of intrinsic dendritic currents and burst generation. We did not study the effects of bicuculline on the amplitude of monosynaptic EPSPs in dendrites. However, somatically recorded EPSPs, which would presumably reflect changes in dendritic EPSPs of CA1 HPCs (Andersen et al., 1980a; Turner and Schwartzkroin, 1980), are not significantly enhanced when slices are exposed to concentrations of hicuculline or penicillin sufficient to induce epileptogenesis (Schwartzkroin and Prince, 1978, 1980). Data such as those of Figure 7 indicate that extremely large EPSPs do not necessarily underlie the burst generation which develops in dendrites after bicuculline (see also Wong and Prince, 1979).

A more likely possibility is that bicuculline is exerting its effects through interference with postsynaptic dendritic GABA receptors that are few, but strategically located. It is possible that the residual axonal arborization of inhibitory interneurons whose somata have been eliminated by the cut, and the small number of inhibitory interneurons normally present in stratum radiatum, continue to release inhibitory transmitter spontaneously or in response to orthodromic stimuli. Bicuculline would depress this dendritic inhibition and facilitate burst generation. Small changes in membrane potential or $R_{N}$, which may result when this presumed low-level inhibition is blocked, might only occur at critical sites on the dendritic arborization and thus go undetected by the intradendritic electrode. The small number of inhibitory synaptic profiles seen in electron micrographs of stratum radiatum (Blackstad and Flood, 1963) implies a "limited" inhibitory input on the apical dendrites. This dendritic inhibition is probably different from the orthodromic or feedforward inhibitory input discussed by Alger and $\mathrm{Ni}$ coll (1982b). The latter is accompanied by hyperpolarization but most likely reflects a recruitment of somatic synapses.

Another possible, but less likely, explanation for the bicuculline effects is the presence of a depolarizing IPSP (Alger and Nicoll, 1982a, b; Wong and Watkins, 1982) on isolated dendrites. If such a potential were present and coincident with the EPSP, it would have gone undetected in our experiments, and its blockade by bicuculline could have enhanced the effectiveness of EPSPs in evoking slow depolarizations and bursts.

In the absence of bicuculline, the effects of dendritic inhibition can potentially be overcome by DC membrane depolarization. This depolarization makes EPSPs effective in reaching threshold for generation of intrinsic dendritic conductances underlying slow depolarizing waves and slow spikes (Fig. $5, A$ and $B$ ). Since it has been shown that significant residual $\mathrm{Cl}^{-}$-mediated IPSPs persist at doses of penicillin which lead to epileptogenic burst discharge (Prince, 1968; Hablitz, 1981), and since our results indicate that burst discharges cannot be evoked at resting membrane potential even when phasic IPSPs have been eliminated by the microdissection, we would speculate that the somatic inhibitory synaptic input is not as crucial in suppressing dendritic burst 
activity as previously proposed. Rather, our data suggest that there exists on isolated apical dendrites of CA1 hippocampal pyramidal cells an inhibitory input that is effective in suppressing burst discharge even in the absence of feedback inhibition. This dendritic inhibition may play a major role in controlling the excitability of CA1 hippocampal pyramidal cells during normal hippocampal activity and may be compromised during epileptogenesis.

Data obtained from isolated dendrites indicate that the effectiveness of dendritic EPSPs in evoking spike discharge may depend in part upon the intrinsically generated slow depolarization of the dendritic membrane, which serves to boost the EPSP amplitude toward the spike threshold, or a level of depolarization at which burst discharges may occur. Synaptically evoked subthreshold dendritic depolarizations were not appreciated in previous studies (Wong et al., 1979; Wong and Prince, 1979); however, our data suggest that such potentials may be prominent under some conditions. Although the addition of a slow depolarizing component to the EPSP at higher orthodromic stimulus intensities (e.g., Fig. $2 C$ ) might be explained as a presynaptic effect such as recruitment of an additional synaptic input, examples in which the EPSP amplitude and duration increased when the membrane potential was made more positive (Figs. $5 B$ and 6) suggest that the EPSP is capable of initiating an intrinsic subthreshold depolarizing event similar to that produced by brief depolarizing current pulses (Fig. 5C, trace 2). In other HPCs, such slow depolarizing events are eliminated by $\mathrm{Ca}^{++}$channel blockers (Wong and Prince, 1978, 1981). These observations, together with results of recent experiments on the electrical properties of isolated dendrites (Benardo et al., 1982), suggest that $\mathrm{Ca}^{++}$may be the charge carrier for synaptically evoked slow dendritic depolarizations in HPCs. A slow, $\mathrm{Ca}^{++}$-dependent depolarizing potential has recently been reported in Purkinje cells, following glutamate iontophoresis onto dendrites (Sugimori and Llinas, 1982), suggesting that synaptically activated subthreshold responses may be a common feature of dendritic membranes. These $\mathrm{Ca}^{++}$-mediated events will affect the amplitude and shape of orthodromic responses recorded at the somatic level and have an important influence on spike generation in dendrites and somata.

\section{References}

Alger, B. E., and R. A. Nicoll (1979) GABA-mediated biphasic inhibitory response in hippocampus. Nature 281: 315-317.

Alger, B. E., and R. A. Nicoll (1982a) Feedforward dendritic inhibition in rat hippocampal pyramidal cells studied in vitro. J. Physiol. (Lond.) 328: 105-123.

Alger, B. E., and R. A. Nicoll (1982b) Pharmacological evidence for two kinds of GABA receptors on rat hippocampal pyramidal cells studied in vitro. J. Physiol. (Lond.) 328: 125-141.

Andersen, P. (1960) Interhippocampal impulses. II. Apical dendritic activation of CA1 neurons. Acta Physiol. Scand. 48: 178-208.

Andersen, P., and T. Lomo (1966) Mode of activation of hippocampal pyramidal cells by excitatory synapses on dendrites. Exp. Brain Res. 2: 217-260.

Andersen, P., J. C. Eccles, and Y. Loyning (1963) Recurrent inhibition in the hippocampus with identification of the inhibitory cell and its synapses. Nature 198: 540-542.

Andersen, P., J. C. Eccles, and Y. Loyning (1964) Pathway of postsynaptic inhibition in the hippocampus. J. Neurophysiol. 27: 608-619.

Andersen, P., T. W. Blackstad, and T. Lomo (1966) Location and identification of excitatory synapses on hippocampal pyramidal cells. Exp. Brain Res. 4: 236-248.

Andersen, P., R. Dingledine, L. Gjerstadt, L. A. Langmoen, J. Masfeldt, and A. Lawsen (1980a) Two different responses of hippocampal pyramidal cells to application of gamma-aminobutyric acid. J. Physiol. (Lond.) 305: 279-296.

Andersen, P., H. Silfvenius, S. H. Sundberg, and O. Sveen (1980b) A comparison of distal and proximal dendritic synapses on CA1 pyramids in guinea-pig hippocampal slices in vitro. J. Physiol. (Lond.) 307: 273-299.

Barrett, J. N., and W. E. Crill (1974) Influence of dendritic location and membrane properties on the effectiveness of synapses on cat motoneurons. J. Physiol. (Lond.) 293: 325345.

Benardo, L. S., L. M. Masukawa, and D. A. Prince (1982) Electrophysiology of isolated hippocampal pyramidal dendrites. J. Neurosci. 2: 1614-1622.

Blackstad, T. N., and P. R. Flood (1963) Ultrastructure of hippocampal axosomatic synapses. Nature 198: 542-543.

Dingledine, R., and L. Gjerstadt (1979) Penicillin blocks hippocampal IPSPs, unmasking prolonged EPSPs. Brain Res. 118: 205-209.

Dingledine, R., and L. Gjerstadt (1980) Reduced inhibition during epileptiform activity in the in vitro hippocampal slice. J. Physiol. (Lond.) 305: 297-313.

Fujita, Y. (1979) Evidence for the existence of inhibitory postsynaptic potentials in dendrites and their functional significance in hippocampal pyramidal cells of adult rabbits. Brain Res. 175: 59-69.

Gottlieb, D. I., and W. M. Cowan (1972) On the distribution of axonal terminals containing spheroidal and flattened synaptic vesicles in the hippocampus and dentate gyrus of the rat and cat. Z. Zellforsch. 129: 413-429.

Hablitz, J. J. (1981) Effects of intracellular injections of chloride and EGTA on postepileptiform-burst hyperpolarizations in hippocampal neurons. Neurosci. Lett. 22: 159-163.

Heyer, E. J., L. M. Nowak, and R. L. MacDonald (1982) Membrane depolarization and prolongation of calcium-dependent action potentials of mouse neurons in cell culture by two convulsants: Bicuculline and penicillin. Brain Res. 232: $41-56$.

Hotson, J. R., and D. A. Prince (1981) Penicillin- and bariuminduced epileptiform bursting in hippocampal neurons: Actions on $\mathrm{Ca}^{+1}$ and $\mathrm{K}^{+}$potentials. Ann. Neurol. 10: 11-17.

Leung, L. S. (1978) Hippocampal CA1 region-demonstration of antidromic dendritic spikes and dendritic inhibition. Brain Res. 158: 219-222.

Llinas, R. (1975) Electroresponsive properties of dendrites in central neurones. Adv. Neurol. 12: 1-13.

Llinas, R., and R. Hess (1976) Tetrodotoxin-resistant dendritic spikes in avian Purkinje cells. Proc. Natl. Acad. Sci. U. S. A. 73: 2520-2523.

Llinas, R., and C. Nicholson (1971) Electrophysiological properties of dendrites and somata in alligator Purkinje cells. J. Neurophysiol. 34: 534-551.

Llinas, R., and M. Sugimori (1980) Electrophysiological properties of in vitro Purkinje cell dendrites in mammalian cerebellar slices. J. Physiol. (Lond.) 305: 197-213.

Llinas, R., C. Nicholson, J. Freeman, and D. E. Ilillman (1968) Dendritic spikes and their inhibition in alligator Purkinje cells. Science 160: 1132-1135.

Llinas, R., R. Baker, and W. Precht (1974) Blockade of inhibition by ammonium acetate action on chloride pump in cat 
trochlear motoneurons. J. Neurophysiol. 27: 522-532.

Lorente de No, R. (1934) Studies on the structure of the cerebral cortex. II. Continuation of the study of the ammonic system. J. Psychol. Neurol. 46: 113-177.

Masukawa, L. M., and D. A. Prince (1982) Enkephalin inhibition of inhibitory input to CA1 and CA3 pyramidal neurons in the hippocampus. Brain Res. 249: 271-280.

Masukawa, L. M., L. S. Benardo, and D. A. Prince (1982) Variation in electrophysiological properties of hippocampal neurons in different subfields. Brain Res. 242: 341-344.

Prince, D. A. (1968) Inhibition in "epileptic" neurons. Exp. Neurol. 21: 307-321.

Purpura, D. P. (1967) Comparative physiology of dendrites. In The Neurosciences, G. C. Quarton, T. Melchuk, and F. O. Schmidt, eds., pp. 372-393, Rockefeller University Press, New York.

Purpura, D. P., R. J. Shofer, and T. Scarff (1965) Properties of synaptic activities and spike potentials of neurons in immature neocortex. J. Neurophysiol. 28: 925-942.

Rall, W. (1967) Distinguishing theoretical synaptic potentials computed for different soma-dendritic distributions of synaptic input. J. Neurophysiol. 30: 1138-1168.

Rall, W. (1977) Core conductor theory and cable properties of neurons. In Handbook of Physiology. Vol. I, Sect. 1: Cellular Biology of Neurons, J. M. Brookhart and V. B. Mountcastle, eds., pp. 39-97, American Physiological Society, Bethesda, MD.

Redman, S. J. (1973) The attenuation of passively propagating dendritic potentials in a motoneurone cable model. J. Physiol. (Lond.) 234: 637-664.

Ribak, C. E., J. E. Vaughn, and K. Saito (1978) Immunocytochemical localization of glutamic acid decarboxylase in neuronal somata following colchicine inhibition of axonal transport. Brain Res. 140: 315-332.

Schwartzkroin, P. A. (1975) Characteristics of CA1 neurons recorded intracellularly in the hippocampal in vitro slice preparation. Brain Res. 85: 423-436.

Schwartzkroin, P. A., and D. A. Prince (1978) Cellular and field potential properties of epileptogenic hippocampal slices. Brain Res. 147: 117-130.
Schwartzkroin, P. A., and D. A. Prince (1980) Changes in excitatory and inhibitory synaptic potentials leading to epileptogenic activity. Brain Res. 183: 61-76.

Spencer, W. A., and E. R. Kandel (1961) Electrophysiology of hippocampal neurons. IV. Fast prepotentials. J. Neurophysiol. 24: 272-285.

Storm-Mathisen, J., and F. Fonnum (1971) Quantitative histochemistry of glutamate decarboxylase in the rat hippocampal region. J. Neurochem. 18: 1105-1111.

Sugimori, M., and R. Llinas (1982) Role of dendritic electroresponsiveness in neuronal integration: In vitro study of mammalian Purkinje cells. Soc. Neurosci. Abstr. 8: 446.

Traub, R. D. (1979) Neocortical pyramidal cells: A model with dendritic calcium conductance reproduces repetitive firing and epileptic behavior. Brain Res. 173: 243-257.

Traub, R. D., and R. Llinas (1979) Ilippocampal pyramidal cells: Significance of dendritic ionic conductances for neuronal function and epileptogenesis. J. Neurophysiol. 42: 476496.

Turner, D. A., and P. A. Schwartzkroin (1980) Steady-state electrotonic analysis of intracellularly stained hippocampal neurons. J. Neurophysiol. 44: 184-199.

Wong, R. K. S., and D. A. Prince (1978) Participation of calcium spikes during intrinsic burst firing in hippocampal neurons. Brain Res. 159: 385-390.

Wong, R. K. S., and D. A. Prince (1979) Dendritic mechanisms underlying penicillin-induced epileptiform activity. Science 204: 1228-1231.

Wong, R. K. S., and D. A. Prince (1981) Afterpotential generation in hippocampal pyramidal cells. J. Neurophysiol. 45: 86-97.

Wong, R. K. S., and D. J. Watkins (1982) Cellular factors influencing the GABA response in hippocampal pyramidal cells. J. Neurophysiol. 45: 938-951.

Wong, R. K. S., D. A. Prince, and A.I. Basbaum (1979) Intradendritic recordings from hippocampal neurons. Proc. Natl. Acad. Sci. U. S. A. 76: 986-990.

Yamamoto, C. (1972) Intracellular study of seizure-like afterdischarges elicited in thin hippocampal sections in vitro. Exp. Neurol. 35: 154-164. 